\title{
Alcohol Related Neurodevelopmental Disorder
}

National Cancer Institute

\section{Source}

National Cancer Institute. Alcohol Related Neurodevelopmental Disorder. NCI Thesaurus.

Code C92728.

A cognitive and neurological disorder due to fetal intrauterine exposure to maternal alcohol consumption. Typically, this presents without facies or other growth abnormalities. 